\title{
Descomposición catalítica del tartrato sódico-potásico
}

Catalytic decomposition of pottasium sodium tatrato

Recibido: agosto 15 de 2017 | Revisado: setiembre 12 de 2017 | Aceptado: noviembre 22 de 2017

Leonardo Félix Machaca Gonzales ${ }^{\mathrm{I}}$

Fabio Manuel Rangel Morales ${ }^{2}$

Carlos Ernesto Ángeles Querolo ${ }^{\mathrm{I}}$ Luis Américo Carrasco Venegas ${ }^{\mathrm{I}}$

\section{RESUMEN}

Se evaluó la descomposición del tartrato sódico-potásico, y su mecanismo de reacción que se usó como catalizador del cloruro de cobalto II el cual mostró su comportamiento en la producción de dióxido de carbono. Asimismo, presentamos la evaluación de los datos experimentales y su mecanismo de reacción detalladamente. Para ello, se han realizado las corridas experimentales con $0,3533 \mathrm{M}$ de tartrato sódico- potásico, peróxido de hidrógeno de 1,7647 M y cloruro de cobalto II de 0,308 M.

Palabras claves: descomposición catalítica, tartrato sódico-potásico

\begin{abstract}
It was evaluated the decomposition of the potassium sodium tatrato and its reaction mechanism that was used as a catalyst for the cobalt chloride II, which showed its perfomance in the production of carbon dioxide. Also we present the evaluation of the experimental data and its reaction mechanism in detail. In order to do this, experimental runs were carried out with $0.35533 \mathrm{M}$ sodiumpotassium tartrate, $1.7647 \mathrm{M}$ hydrogen peroxide and $0.308 \mathrm{M}$ cobalt II chloride.
\end{abstract}

Key words: Catalytic decomposition, potassium sodium tatrato
1 Universidad Nacional del Callao clave012@yahoo.es 


\section{Introducción}

Los procesos de transformación industrial involucran procesos catalíticos y no catalíticos. Los procesos catalíticos, a su vez, incluyen a los procesos catalíticos, homogéneos y heterogéneos. En la industria química, los catalizadores son muy importantes debido a que su empleo aumenta la eficiencia del proceso de transformación química o disminuyen el coste total de producción (Langmuir, 1916).

En el proceso de transformación química, existe una etapa elemental, la cual se caracteriza por tener una velocidad menor que la del resto de etapas que limita la velocidad global de la reacción del proceso químico. Para conseguir que la reacción transcurra, a mayor velocidad, una opción sería aumentar la temperatura; sin embargo, esto podría disminuir la concentración en el equilibrio del producto que deseamos o bien producirse reacciones secundarias que consuman el producto deseado o que generen impurezas. Para evitar todo esto suele ser útil la adición de un catalizador, conociéndose como reacciones químicas catalizadas (Vitoria, 1946).

Existen reacciones catalizadas en las que el catalizador sufre algún tipo de transformación, por ejemplo, una descomposición, y esta transformación es independiente de la reacción principal. Por otro lado, los catalizadores que disminuyen la velocidad de la reacción se les conoce como inhibidores o catalizadores negativos, y su aplicación industrial más importante es la reducción de reacciones secundarias hacia productos no deseados (Vitoria, 1946). Por lo tanto, los catalizadores son muy importantes en la industria química, donde su uso puede aumentar la eficiencia de un proceso químico o disminuir el coste total al fabricante. No resulta sorprendente, por tanto, que se haya dedicado mucho tiempo y considerables investigaciones al descubrimiento de nuevos y mejores catalizadores (Langmuir, 1916). A pesar de ello, no se conocen bien los mecanismos de muchas reacciones catalizadas, y muchos catalizadores utilizados en la industria química, se descubrieron mediante tanteo más que por investigación (Matín-Sánchez, 1986).

La adición de un catalizador no afecta, directamente, a la termodinámica de la reacción. El papel de un catalizador es acelerar las reacciones directa e inversa en la misma proporción y como consecuencia, reducir el tiempo que se tarda en alcanzar el equilibrio. Así, en presencia de un catalizador, es posible utilizar, a menudo, condiciones experimentales diferentes de modo que la reacción transcurra más rápidamente, incluso aunque las condiciones termodinámicas no sean las adecuadas. Asimismo, la presencia del catalizador no altera la constante de equilibrio, pero se incrementa la velocidad con que se alcanza. El papel del catalizador se observa en el diagrama energía potencial-coordenada o transcurso de la reacción, y la dependencia de las velocidades de reacción con un catalizador que se muestra en la Figura 1 (García-Liarte \& Peña-Martínez, 2010).

$\mathrm{Si}{E_{1}}_{+}^{+} \mathrm{y}_{E_{-1}+}^{+}$son las energías de activación de las reacciones directa e inversa respectivamente, el catalizador reduce la altura de la barrera de energía de activación en un valor $\mathrm{E}_{\mathrm{A}}$. 
La adición de una sustancia denominada veneno del catalizador o un inhibidor decrece la velocidad de una reacción química. A menudo, se utiliza la denominación catalizador negativo, pero es engańosa ya que dicho aditivo se consume a veces en la reacción. El inhibidor puede hacer disminuir la velocidad de una reac- ción al originar un proceso competitivo con el reactivo (Blanco \& Linares, 1976).

Este trabajo trata del estudio de la descomposición del tartrato sódico potásico conocido como sal de Rochelle con peróxido de hidrógeno usando al cloruro de cobalto como catalizador.

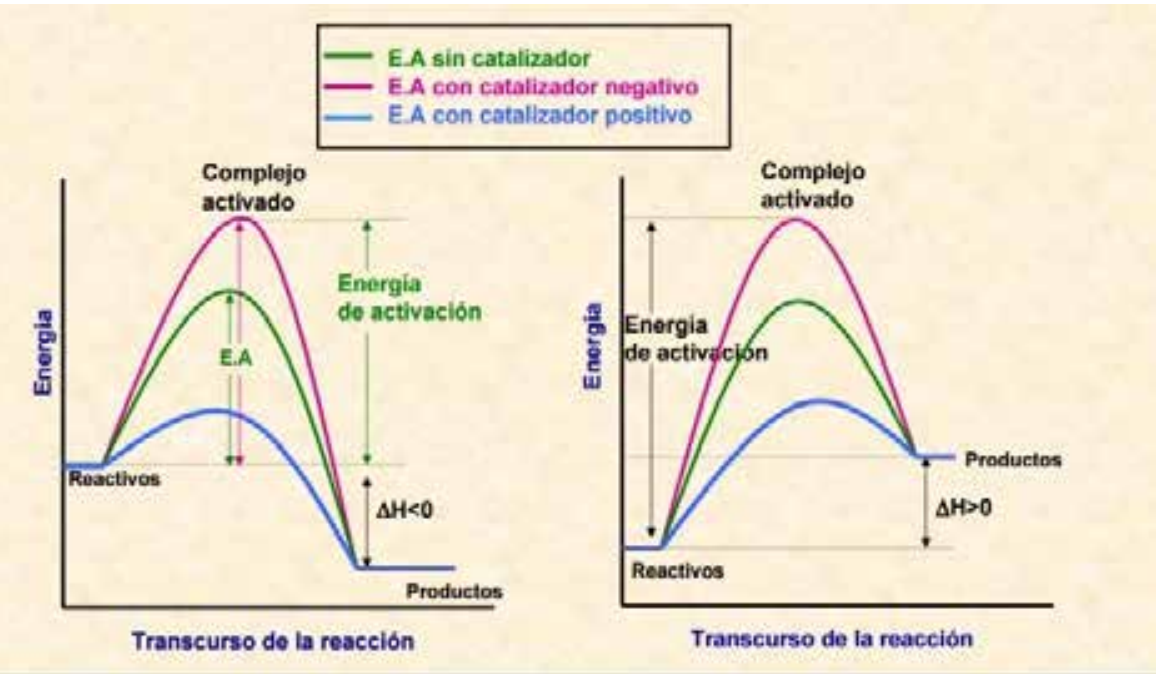

Figura 1. Diagrama de energía potencial mostrando el descenso de la barrera de energía de activación para una reacción catalizada. Fuente: Avery (1974).

\section{Fundamento teórico}

Las reacciones catalíticas homogéneas presentan la particularidad de proceder a bajas temperaturas con selectividades elevadas. Esto se logra mediante la selección adecuada del metal de transición, de los líquidos, del solvente, y de las condiciones de reacción. Este es el reflejo del conocimiento mecánico que caracteriza a la catálisis homogénea y que permite optimizar el proceso al conocer los ciclos catalíticos completos (Langmuir, 1916).

Las catálisis homogéneas, en fase líquida, son frecuentes. Dentro de ellas, las más investigadas han sido las catálisis por iones, principalmente, las catálisis ácido y base, que incluyen la esterificación, sa- ponificación, inversión, mutarrotación, enolización y muchas oxidaciones y reducciones (Sabatier, 2007).

Así, los ácidos y las bases se relacionan por la ecuación (Bell, 1941):

$$
\text { Ácido } \rightleftharpoons \text { base }+H^{+}
$$

Las sales neutras, en disolución, influyen sobre la reactividad. Lógicamente, las colisiones de los iones están influidas por la atmósfera iónica que, a su vez, vienen afectadas por la presencia de sales (Sabatier, 2007).

Muchas reacciones homogéneas en disolución son catalizadas por ácido y bases. La hidrólisis de un éster es un ejemplo bien conocido de reacción que 
es catalizada tanto por los ácidos como las bases. La mutarrotación de la glucosa es un ejemplo de reacción catalizada por ácido, bases y disolventes (Sabatier, 2007).

Avery (1974) considera un sustrato $S$ que experimenta una reacción elemental con un ácido, una base o con ambos. Asimismo, menciona que la definición de Lowry-Bronsted de ácidos y bases establece un ácido como una sustancia que cede un protón.

$$
\mathrm{HA}+\mathrm{H}_{2} \mathrm{O} \rightarrow \mathrm{H}_{3} \mathrm{O}^{+}+\mathrm{A}^{-}
$$

y una base como una sustancia que acepta un protón

$$
\mathrm{A}^{-}+\mathrm{H}_{2} \mathrm{O} \rightarrow \mathrm{HA}+\mathrm{OH}^{-}
$$

La velocidad de una reacción catalizada viene dada por: $v=k_{\text {cat }}[S]$ donde: $\mathrm{k}_{\text {cat }}$ es un coeficiente catalítico de velocidad. Por consiguiente:

$$
\begin{aligned}
k_{c a t}= & k_{0}+k_{H^{+}}\left[\mathrm{H}_{3} \mathrm{O}^{+}\right]+k_{O H^{-}}\left[\mathrm{OH}^{-}\right] \\
& -k_{H A}[\mathrm{HA}]+k_{A^{-}}\left[A^{-}\right]
\end{aligned}
$$

Donde: $k_{0}$ es el coeficiente de velocidad para la reacción no catalizada, $y, \mathrm{k}_{\mathrm{H}^{+}}$, $\mathrm{k}_{\mathrm{OH}^{-}}$, $\mathrm{k}_{\mathrm{HA}} \mathrm{y} \mathrm{k}_{\mathrm{A}^{-}}$son constantes de velocidad catalítica para las especies indicadas.

En la catálisis ácido-base específica, hay algunas reacciones en que la velocidad es proporcional solo a la concentración de los iones $\mathrm{H}_{3} \mathrm{O}^{+} \mathrm{y} \mathrm{OH}^{-}$presentes. Tales reacciones son ejemplos de catálisis específica ácido-base. En esta situación una o ambas de las constantes son grandes com- paradas con las constantes y $k_{H A}$ y $k_{A^{-}}$ la ecuación 4 se reduce a (Avery, 1974):

$$
\mathrm{k}_{\mathrm{cat}}=\mathrm{k}_{0}+\mathrm{k}_{\mathrm{H}^{+}}\left[\mathrm{H}_{3} \mathrm{O}^{+}\right]+\mathrm{k}_{\mathrm{OH}^{-}}\left[\mathrm{OH}^{-}\right](5)
$$

Si la reacción solo es catalizada por los ácidos como en la inversión del azúcar.

$$
k_{\text {cat }}=k_{0}+k_{\mathrm{H}^{+}}\left[\mathrm{H}_{3} \mathrm{O}^{+}\right]
$$

En forma similar, para una reacción catalizada solo por las bases

$$
k_{c a t}=k_{0}+k_{O^{-}}\left[\mathrm{OH}^{-}\right]
$$

El coeficiente catalítico de velocidad se determina midiendo la velocidad de una disolución de fuerza iónica constante en un amplio intervalo de $\mathrm{pH}$ con disoluciones reguladoras apropiadas (Avery, 1974; Sabatier, 2007).

Para una reacción con una concentración elevada de ácido de modo que la ecuación 4 se convierte en:

$$
k_{\text {cat }}=k_{H^{+}}\left[\mathrm{H}_{3} \mathrm{O}^{+}\right]
$$

Tomando logaritmos se tiene:

$$
\begin{gathered}
\log _{10} k_{\text {cat }}=\log _{10} k_{H^{+}} \log _{10}\left[\mathrm{H}_{3} \mathrm{O}^{+}\right] \\
\log _{10} k_{\text {cat }}=\log _{10} k_{\mathrm{H}^{+}}-\mathrm{pH}
\end{gathered}
$$

En el intervalo ácido de $\mathrm{pH}$, la representación de $\log _{10} k_{c a t}$ frente al $\mathrm{pH}$ será una línea recta de pendiente igual a -1 .

En una disolución fuertemente básica, la ecuación (7) se transforma en $k_{\text {cat }}=k_{\mathrm{OH}^{-}} \quad\left[\mathrm{OH}^{-}\right]=k_{\mathrm{OH}^{-}} \frac{K_{w}}{\left[\mathrm{H}_{3} \mathrm{O}^{+}\right]}$

donde $K_{w}$ es el producto iónico del agua.

Tomando logaritmos

$$
\log _{10} k_{c a t}=\log _{10} k_{O H^{-}} \quad k_{w}+p H
$$




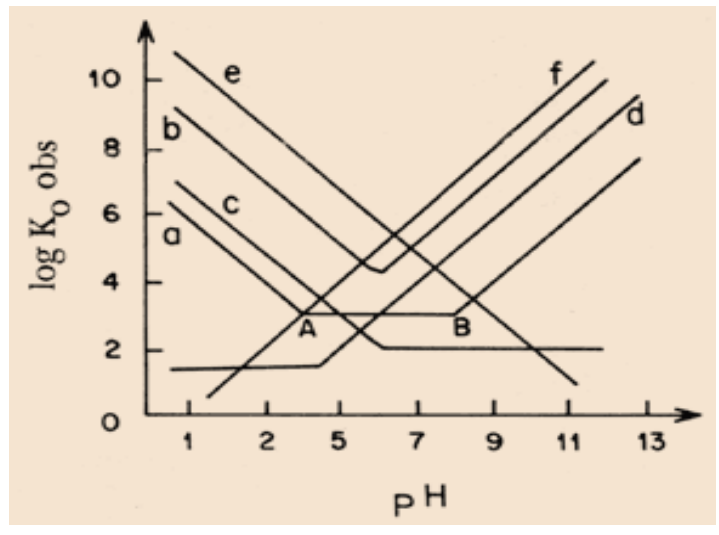

Figura 2. Efecto del $\mathrm{pH}$ en la velocidad de algunas reacciones catalizadas mediante ácidos o bases.

Una representación de $\log _{10} k_{\text {cat }}$ en función del $\mathrm{pH}$ será, por tanto, lineal con pendiente 1 (Figura 2). En la región intermedia de $\mathrm{pH}, \log _{10} k_{\text {cat }}$ llega a hacerse independiente de $\left[\mathrm{H}_{3} \mathrm{O}^{+}\right]$y $\left[\mathrm{OH}^{-}\right]$. En dichos casos depende solo de $k_{0}$ es independiente del $\mathrm{pH}$ (Avery, 1975).

Mientras que en la catálisis general ácido-base, las reacciones que son catalizadas por todos los ácidos y bases de Lowry-Bronsted presentes en la disolución se dice que presentan catálisis general ácido-base. Cuando la disolución está regulada tal que la velocidad no sea afectada por los iones $\mathrm{H}_{3} \mathrm{O}^{+}$y $\mathrm{OH}^{-}$-esto es, $k_{H+} y k_{O H-}$ son despreciables y se mantiene constante la fuerza - la velocidad de muchas reacciones depende de la concentración de ácido indisociado HA y de la concentración de la base conjugada A. Suponiendo que la reacción no catalizada representa una contribución despreciable a la velocidad, tenemos(Avery, 1974; Sabatier, 2007):

$$
k_{\text {cat }}=k_{H A}[H A]+k_{A^{-}}[A]
$$

La reacción se estudia a dos valores del $\mathrm{pH}$ para cada uno de los cuales $[H A] /\left[A^{-}\right]$es una constante conocida, que podemos llamar $\mathrm{x}_{1} \mathrm{y} \mathrm{x}_{2}$ respectivamente. Bajo esas condiciones tenemos:

$$
\begin{aligned}
& k_{\text {cat }}=k_{H A}[H A]+k_{A^{-}} \frac{[H A]}{x_{1}} \\
& k_{\text {cat }}=k_{H A}[H A]+k_{A^{-}} \frac{[H A]}{x_{2}}
\end{aligned}
$$

El $\mathrm{CoCl}_{2} \cdot 6 \mathrm{H}_{2} \mathrm{O}$ y $\mathrm{CoCl}_{2}$ son ácidos de Lewis débiles que forman muchos otros compuestos coordinados. Estos complejos de cobalto (II) son, normalmente, octaédricos o tetraédricos (Avery, 1974; Sabatier, 2007; Vitoria, 1946).

Existen varios métodos para evaluar los parámetros cinéticos de la velocidad de reacción, así podemos como (Avery, 1974; García-Lairte \& Peña-Martínez, 2010):

1. El método dilatómetro se usa cuando una reacción implica cambio de volumen. Dicho cambio es directamente proporcional al grado de avance de la reacción, podemos mencionar el caso de la reacción de la hidrólisis del acetal en caserío de agua, catalizada por los ácidos.

$$
\begin{gathered}
\mathrm{CH}_{3} \mathrm{CH}\left(\mathrm{OC}_{2} \mathrm{H}_{5}\right)_{2}+\mathrm{H}_{2} \mathrm{O} \stackrel{\left[\mathrm{H}^{+}\right]}{\longrightarrow} \\
\mathrm{CH}_{3} \mathrm{CHO}+2 \mathrm{C}_{2} \mathrm{H}_{5} \mathrm{OH}
\end{gathered}
$$

Esta reacción (ecuación 16) produce un aumento de volumen que puede medirse mediante un dilatómetro. Este consiste en un matraz de reacción al que se conecta un capilar y uniforme con una escala calibrada. La variación de nivel del líquido en el capilar se lee utilizando un catetómetro y se determina el cambio de volumen.

Si $V_{0}, V_{\infty}$ y $V_{t}$, corresponden a los volúmenes inicial, final y al del tiempo $t$, respectivamente, la ecuación de primer orden viene dada por: 


$$
\begin{gathered}
\log _{10}\left(V_{\infty}-V_{t}\right)= \\
\log _{10}\left(V_{\infty}-V_{0}\right)-\frac{k_{v} t}{2,303}
\end{gathered}
$$

Un representación de $\log _{10}\left(V_{\infty}-V_{t}\right)$ es función de $t$ será lineal con pendiente $-k v / 2,303$.

2. El método del desprendimiento gaseoso se usa para una reacción en disolución en la cual uno de los productos es un gas. Podemos mencionar para este método la reacción del cloruro de bencenodiazonio que se descompone en disolución acuosa a temperatura ambiente liberando nitrógeno de acuerdo con la ecuación:

$$
\begin{aligned}
& \mathrm{C}_{6} \mathrm{H}_{5} \mathrm{~N}_{2} \mathrm{Cl}^{-}+\mathrm{H}_{2} \mathrm{O} \rightarrow \\
& \mathrm{C}_{6} \mathrm{H}_{5} \mathrm{OH}+\mathrm{HCl}+\mathrm{N}_{2}
\end{aligned}
$$

En exceso de agua, la reacción es de primer orden y obedece a la ecuación de velocidad (Avery, 1974; Sabatier, 2007).

$$
k_{v} t=2,303 \log _{10}\left(\frac{a}{a-x}\right)
$$

Sea $V_{\infty}$ el volumen de nitrógeno desprendido cuando la reacción se ha completado, y sea $\mathrm{V}_{\mathrm{t}}$ el volumen desprendido en el tiempo $t$. Dado que $a$ es proporcional a $V_{\infty}$ y $x$ es proporcional a $V_{t}$ tenemos:

$$
\begin{gathered}
k_{v} t=2,303 \log _{10}\left(\frac{V_{\infty}}{V_{\infty}-V_{t}}\right) o \\
\log _{10}\left(V_{\infty}-V_{t}\right)=\log V_{\infty}-\frac{k_{v} t}{2,303}
\end{gathered}
$$

La representación de $\left(V_{\infty}-V_{t}\right)$ en función de $t$ es lineal con pendiente $-\mathrm{kv} / 2,303$.

\section{Materiales y métodos}

Para evaluar la velocidad de descomposición del tartrato de sodio y potasio, fue necesario seguir el desprendimiento del gas de $\mathrm{CO}_{2}$ producido con respecto al tiempo.

En este experimento, se ha utilizado el método desprendimiento gaseoso para evaluar experimentalmente la reacción de descomposición catalítica de tartrato de sodio y potasio y su mecanismo de reacción (Figura 3).

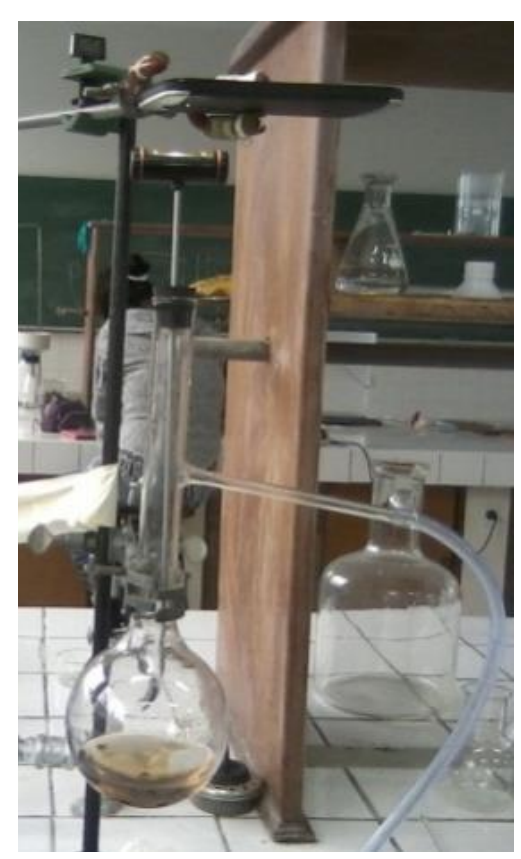

Figura 3. Arreglo experimental

\section{Desarrollo experimental}

El desarrollo experimental nos ha permitido determinar el efecto del catalizador sobre la velocidad de descomposición catalítica del tartrato sódico midiendo el volumen del agua desplazada por el gas de $\mathrm{CO}_{2}$ producido en función al tiempo, tal como se muestra en Figura 3. 
Se prepararon soluciones de:

- Tartrato de sodio y potasio de concentración $0,35 \mathrm{M}$ disolviendo $5 \mathrm{~g}$ en $50 \mathrm{ml}$ de agua destilada.

- Cloruro de cobalto de concentración $0,31 \mathrm{M}$ disolviendo $0,2 \mathrm{~g}$. en $5 \mathrm{ml} \mathrm{de}$ agua.

- Peróxido de hidrógeno 20 volúmenes, de concentración 1,76 M.

El tartrato de sodio y potasio se vierte al reactor conjuntamente con el peróxido de hidrógeno y añade la solución de cloruro de cobalto que actúa como catalizador. Estas soluciones se agitan suavemente para la homogenización y la temperatura de inicio es de $19,6^{\circ} \mathrm{C}$.

Finaliza la reacción cuando deja de producirse el dióxido de carbono, que indica que ya no hay tartrato, y aparece de nuevo el color rosa de la sal de cobal- to. De esta forma, se comprueba que el catalizador no se gasta en la reacción.

El gas de $\mathrm{CO}_{2}$ desprendido, desplaza al agua de la manguera instalada en forma de serpentín desde el reactor hacia la bureta graduada y se ha medido el volumen de dicho gas a intervalos de tiempo adecuados, que se muestra en los resultados y la temperatura de la reacción alcanza hasta $56^{\circ} \mathrm{C}$, comprobándose que es una reacción exotérmica.

\section{Resultados}

Se obtuvieron los resultados que se muestran en la Figura 4.

En este mecanismo, ocurren en forma simultánea, la oxidación y reducción del ion tartrato con el peróxido de hidrógeno y también la oxidación del ion cobalto, tal como se ilustra:

\section{Mecanismo de la reacción de descomposición del tartrato sódico potásico}

\section{Reacción no catalizada:}

$\mathrm{C}_{4} \mathrm{H}_{4} \mathrm{O}_{6}-2+5 \mathrm{H}_{2} \mathrm{O}_{2} \stackrel{k_{1}}{\rightarrow} \mathrm{CO}_{2}+6 \mathrm{H}_{2} \mathrm{O}+2 \mathrm{OH}^{-}$

\section{Reacción catalizada:}

Etapa 1. Reacción de formación del complejo activado (reacción reversible):

$$
\begin{array}{ccc}
\mathrm{C}_{4} \mathrm{H}_{4} \mathrm{O}_{6}{ }^{-2}+2 \mathrm{OH}^{-}+\mathrm{Co}^{+2} & \stackrel{\mathrm{K}_{2}}{\Leftrightarrow} & \left(\mathrm{C}_{2} \mathrm{H}_{2} \mathrm{O}_{3}{ }^{-2}\right)_{2}>\mathrm{Co}^{+3} \\
\text { Rosáceo } & (\mathrm{OH})_{2} \\
& & \text { verde oscuro }
\end{array}
$$

Etapa 2. Reacción de descomposición del complejo activado (reacción irreversible):

$$
\left(\mathrm{C}_{2} \mathrm{H}_{2} \mathrm{O}_{3}^{-2}\right)_{2}>\mathrm{Co}^{+3} .(\mathrm{OH})_{2}
$$

Verde oscuro

$$
\stackrel{k_{3}}{\Rightarrow} 4 \mathrm{CO}_{2}+6 \mathrm{H}_{2} \mathrm{O}+2 \mathrm{OH}^{-}+\mathrm{Co}^{+2}
$$

rosáceo 


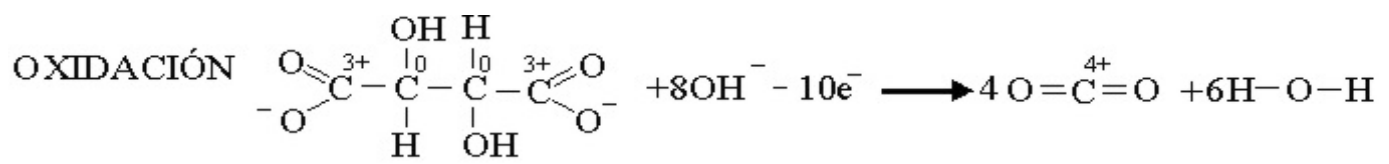

$$
\begin{aligned}
& \text { REDUCCIÓN } \quad 5 \mathrm{H}^{-} \stackrel{1-}{\mathrm{O}-}-\mathrm{O}^{-}-\mathrm{H}+10 \mathrm{e}^{-} \longrightarrow 10 \mathrm{OH}^{-}
\end{aligned}
$$

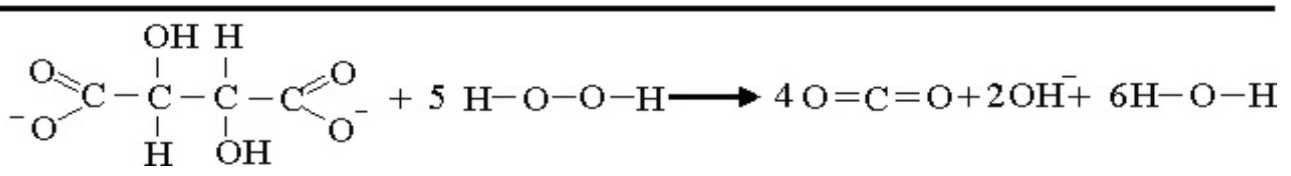

$\mathrm{Al}$ estar con el contacto el $\mathrm{Co}^{+2}$ con peróxido de hidrógeno se oxida a $\mathrm{Co}^{+3}$ (verde oscuro), según el mecanismo posible:

$$
\begin{aligned}
& \text { Reducción: } \mathrm{H}-\mathrm{O}-\mathrm{O}-\mathrm{H}+2 \mathrm{e}^{-} \rightarrow 2 \mathrm{OH}^{-} \\
& \text {Oxidación: } 2 \mathrm{Co}^{+2}-2 \mathrm{e}^{-} \rightarrow 2 \mathrm{Co}^{+3} \text { (verde oscuro) } \\
& \qquad \begin{array}{l}
\mathrm{H}-\mathrm{O}-\mathrm{O}-\mathrm{H}+2 \mathrm{Co}^{+2} \rightarrow 2 \mathrm{OH}^{-}+2 \mathrm{Co}^{+3}
\end{array}
\end{aligned}
$$

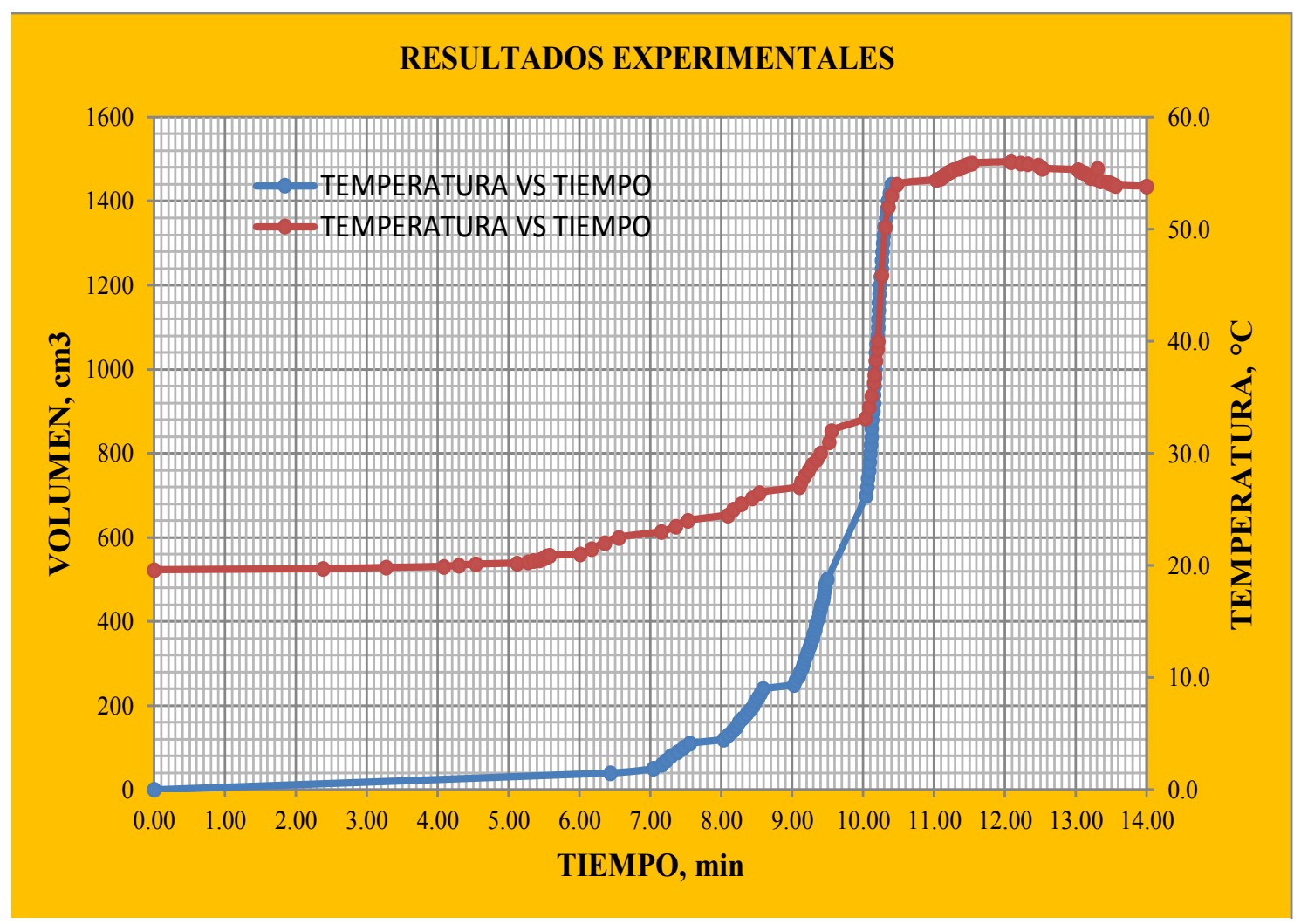

Figura 4. Gráfico de los resultados experimentales de la descomposición catalítica del tartrato de sodio y potasio

\section{Discusión}

La Figura 4 nos indica que la reacción de descomposición catalítica sigue la for- ma del crecimiento bacteriano: una biorreacción. Hay descomposición lenta del tartrato de sodio y potasio; es decir, se ha 
producido un volumen de gas de $\mathrm{CO}_{2}$ de $50 \mathrm{ml}$ en siete minutos. Enseguida, la descomposición del tartrato va en aumento moderado desde los siete hasta 10 minutos, y la temperatura en ese mismo intervalo de tiempo desde $19,8{ }^{\circ} \mathrm{C}$ hasta $35,1^{\circ} \mathrm{C}$, produciéndose $700 \mathrm{ml}$. Luego la descomposición del tartrato de sodio y potasio aumenta, bruscamente, en el intervalo desde 10 hasta 10,40 minutos, produciéndose $1440 \mathrm{ml}$ de gas de $\mathrm{CO}_{2}$. Es decir, en 40 segundos se producen $740 \mathrm{ml}$ de gas de $\mathrm{CO}_{2}$. Finaliza la reacción con un descenso de temperatura desde $56^{\circ} \mathrm{C}$ hasta $53,8^{\circ} \mathrm{C}$.El mecanismo de reacción de descomposición del tartrato se demuestra la secuencia de una reacción catalítica de la forma:
Reacción no catalítica

$$
\mathrm{A}+\mathrm{B} \rightarrow \mathrm{R}+\mathrm{S}
$$

Reacción catalítica

$$
\begin{aligned}
\mathrm{A}+\mathrm{B}+\mathrm{C} \leftrightarrow \mathrm{ABC}^{*} \\
\mathrm{ABC}^{*} \rightarrow \mathrm{R}+\mathrm{S}+\mathrm{C}
\end{aligned}
$$

\section{Conclusiones}

Se ha determinado, experimentalmente, la reacción de descomposición del tartrato de sodio y potasio en medio de la solución de peróxido de hidrógeno por el efecto del cloruro de cobalto que actúa como catalizador, siendo la sustancia que aumenta la velocidad de reacción de un reactivo, pero no altera la estequiometría de este mismo, asimismo, el mecanismo de reacción.

\section{Referencias}

Bell, R. P. (1941). Acid-Base catalysis. England: Clarendon Press Oxford.

Sabatier, P. (2007). Catalysis in Organic Chemistry. Case Press.

Vitoria, E. (1946). La catálisis en Química (4a ed.). Barcelona, España: Casals.

Martín-Sánchez, M.T. (1986). Trabajos Experimentales en una Clase de Química de Nivel Elemental (Documento nro. 12). España: Instituto de Ciencias de la Educación, Universidad de Salamanca.

Blanco, J. \& Linarte, R. (1976). Catálisis: fundamentos y aplicaciones industriales. México: Trillas.
Avery, H.E. (1974). Basic Reaction Kinetics and Mechanisms. England: MacMillan.

García-Lairte, D. A. \& Peña-Martínez, M. (2010). Cinética recreativa. Un análisis de aspectos relativos a la velocidad de las reacciones quimicas basado en el desarrollo de experiencias de gran impacto visual. II Jornada sobre la enseñanza de las ciencias y las ingenierías en la Universidad de Murcia, España.

Langmuir, I. (1916). The constitution and fundamental properties of solids and liquids. Journal of the American Chemical Society, 38(11), 22212295. doi: $10.1021 /$ ja02268a002 